\author{
ANNALES \\ POLONIOI MATHEMATICI \\ $\mathrm{XXV}$ (1872)
}

\title{
Partitions of spectral sets
}

\author{
by W. MLaK (Kraków)
}

Let $H$ be a complex Filbert space with the inner product $(f, g)(f, g \in H)$ and the norm $\|f\|=\sqrt{(f, f)} . L(H)$ stands for the algebra of all linear bounded operators in $H$. For $T \in L(H), T^{*}$ is the adjoint of $T$ and $T \mid S$ is the restriction of $T$ to the invariant subspace $S$. $\|T\|$ is the norm of $T \epsilon L(H) . I$ stands for the identity operator.

Suppose $X$ is a compact Hausdorff space. Let $A$ be a function algebra on $X$, i.e. a uniformly closed subalgebra of $O(X)$, containing constants and separating points of $X$.

The algebra homomorphism $T: A \rightarrow I(H)$ is called a representation of $A$. The representation $T$ is called a $K$-representation if

$$
\|T(u)\| \leqslant K\|u\| \quad\left(\|u\|=\sup _{\bar{X}}|u|\right) \quad \text { for } u \in \mathcal{A} .
$$

If $K=1$, then $T$ is a contractive representation. Without any loss of generality we assume that

$$
T(1)=I .
$$

Given the $K$-representation $T: A \rightarrow L(H)$ there are regular Borel measures $p(f, g)(f, g \in H)$ on $X$ such, that

$$
\begin{array}{cl}
\|p(f, g)\| \leqslant K\|f\|\|g\| & (f, g \in H), \\
(T(u) f, g)=\int_{\mathcal{X}} u d p(f, g) ; & u \in A ; f, g \in H .
\end{array}
$$

Suppose closed $\sigma \subset X$. We say that the $K$-representation $T: A \rightarrow L(H)$ is $\sigma$-supported if it has a system of elementary measures vanishing outside $\sigma$. Write $A_{\sigma}$ for the uniform closure (in $C(\sigma)$ ) of $A \mid \sigma$. If $T$ is a $\sigma$-supported $K$-representation of $A$, then there is a unique representation $T_{\sigma}: A_{\sigma}$ $\rightarrow L(H)$ such that $T(u)=T_{\sigma}(u \mid \sigma)$ for $u \in A$. $T_{\sigma}$ has $\sigma$-elementary measures.

Let $P_{\epsilon} L(H)$ be a projection $\left(P=P^{2}\right.$ !) which commutes with all $T(u)$, where $T: A \rightarrow L(H)$ is a $K$-representation of $A$. Define $H^{\prime}=P H$, 
$H^{\prime \prime}=(I-P) H$. Then $T^{\prime}(u)=T(u)\left|H^{\prime}, T^{\prime \prime}(u)=T(u)\right| H^{\prime \prime}$ define continuous representations of $A ; H=H^{\prime}+H^{\prime \prime}$ and $T=T^{\prime}+T^{\prime \prime}$, both sums direct.

Assume $\sigma \subset X$ is a peals set for $A$. Then $A_{\sigma}=A \mid \sigma$ by a result of [7]. If $p(f, g)$ are $X$-elementary measures of the $K$-representation $T: A \rightarrow L(H)$, then by [12] there is a unique projection $P_{\sigma}$, such that

$$
\begin{gathered}
P_{\sigma} T(u)=T(u) P_{\sigma} \quad \text { for } u \in A, \\
\left(P_{\sigma} T(u) f, g\right)=\int_{X} u \chi_{\sigma} d p(f, g), \quad u \in A ; f, g \in H .
\end{gathered}
$$

$\chi_{\sigma}$ is the characteristic function of $\sigma$. It follows that $T_{\sigma}(u) \stackrel{\text { df }}{=} T(\tilde{u}) \mid P_{\sigma} H$ $(u=\tilde{u} \mid \sigma, \tilde{u} \in A)$ is a $K$-representation of $A_{\sigma}$ in $P_{\sigma} H$. We call it the $\sigma$-part of $T$.

Suppose we are given a compact set $X \subset C$. Let $R(X)$ be the closure in $O(X)$ of restrictions to $X$ of the algebra of rational functions having poles off $X . X$ with the natural topology of the complex plane may be identified with the spectrum of $R(X)$ and $\partial X$, the topological boundary of $X$, with the Shilov boundary of $R(\dot{X})$.

Let $u_{1}(z) \stackrel{\text { dt }}{=} z$ and let $T$ be a $K$-representation of $R(X)$. It follows that the spectrum $\operatorname{sp}(V)$ of $V \stackrel{d f}{=} T\left(u_{1}\right)$ is in $X$. Conversely, if for some $\nabla \in L(H), \operatorname{sp}(V) \subset \bar{X}$, and

$$
\|u(V)\| \leqslant K \sup _{X}|u|
$$

for rational $u$ with poles off $X$, then the representation $u \rightarrow u(V)$ extends uniquelly to a $K$-representation of $R(X)$. We then say that $V$ generates ' this representation and that $X$ is a $K$-spectral set of $V$. This definition extends the one of von Neumann [15] and already has appeared in literature - see [2] for example. If $K=1$ we simply say that $X$ is a spectral set of $V$ - this is the von Neumann definition.

If $R(X)$ is viewed as a function algebra on $X$, then for every its peals set $\sigma \subset X$ we have $R(X) \mid \sigma=R(\sigma)-$ see [1]. We infer from the preceding story that the following theorem holds true:

THEOREM 1. Let the oompact $X \subset C$ bo a $K$-spectral set of $V_{\in} L(H)$. If $\sigma \subset X$ is a peate set of $R(X)$ and $T_{\sigma}$ is the $\sigma$-part of the representation generated by $V$, then $V_{\sigma}=T_{\sigma}\left(u_{1}\right)=V \mid P_{\sigma} H$. has $\sigma$ as a $K$-spoctral sot.

COROLLARY 1. Supposo $\sigma$ is a peate set of $R(X)$ and $X$ is $K$-speotral for $V \in L(H)$. Let $P_{\sigma}$ be the corresponding projection and define an operator valued measure $I F$ on the ring generated by $\sigma$ and $X-\sigma$ as follows:

$$
F(\mathscr{C})=0, \quad F(\mathcal{X})=I, \quad F(\sigma)=P_{\sigma}, \quad F(X-\sigma)=I-P_{\sigma},
$$


Ff is a speotral measure, i.e. $F\left(\sigma_{1}\right) . F\left(\sigma_{9}\right)=F\left(\sigma_{1} \cap \sigma_{2}\right)$. It follows from a theorem of $S z .-N a g y$-Dixmier [3], that there is an $S \in L(H)$, with inverse $S^{-1} \in L(H)$ such that

$$
E(\beta)=\$ F(\beta) S^{-1}
$$

is an orthogonal measure. We infer now from Theorem 1 that $V$ is similar to a sum $\nabla_{1} \oplus V_{2}$, where $V_{1}$ has $\sigma$ as a $K_{1}$-spectral set with $K_{1}=\|K\|\|S\|\left\|S^{-1}\right\|$ and $V_{2}$ generates an $X-\sigma$ supported oontinuous representation of $R(X)$. If $\sigma$ is an interpolation pealc set, i.e. if $R(X) \mid \sigma=O(\sigma)$, then again by $\mathbf{S z}$. Nagy-Dixmiar theorem $V_{I}$ is similar to a normal operator. Thi is a slight axtansion of a result of Douglas ([2], Th. 4).

If $\Pi=1$, then simply $\nabla=V_{1} \oplus \nabla_{2}$.

CoRollary 2. If $\sigma$ is a maximal set of antisymmetry for $R(X)$, then $\sigma$ is a peak set for $R(X)$ by the results of Glicksberg [7]. It follows that if $X$ is $K$-spectral for $V \in L(H)$, then $\sigma$ is $K$-spectral of $V_{\sigma}=\nabla \mid P_{\sigma} H$.

Assume the compact $X \subset C$ is $K$-spectral for $\nabla \in L(H)$ and let $X=\bigcup_{k \mid 0}^{s} X_{l_{k}}(s \leqslant \infty)$ be the Bishop decomposition of $X$ relative to $R(X)$. $X_{1}, X_{2}, \ldots$ are peals sets with positive planar Lebesgue measures and $X_{0}$ is a union of peals point maximal sets of antisymmetry. For details we refer to [7] and [1]. Using the theorems of [12] we get by Theorem 1 that there is a sequence of projections $P_{0}, P_{1}, P_{2}, \ldots$ commuting with $V$ such that $V_{k}=\nabla \mid P_{k} H(k=1,2, \ldots)$ has $X_{k}$ as a $K$-spectral set. Moreover, $V_{0}=V \mid P_{0} H$ has $X$ as a $\pi$-spectral set with $X_{0}$-supported elementary measures and $I=\sum_{k \mid 0}^{s} P_{k}$ wealkly. If $K=1$, then $I=\stackrel{s}{\oplus} P_{k \mid 0}$ and $\nabla=\stackrel{s}{\oplus} V_{k}$.

In what follows we need the following proposition which belongs to I. Glicksberg ( $\left.{ }^{1}\right)$

Proposition 1 . If $p \perp R(X)$, then $p$ vanishes identically on $X_{0}$.

Proof. Since there are no completely singular orthogonal measures for $R(\bar{X})$ ([18], Th. 3.2), the Corollary 1.3 of [8] yields that $p=\sum_{n} p_{n}$, where the measures $p_{n} \ll M_{z_{n}}$ with $z_{n}$ running over non-trivial Gleason parts for $R(X)$, hence carried by $X_{k}$ with $l_{i}>0$. So $p$ is carried by $\bigcup_{k \mid 1}^{s} X_{k}$. which proves the assertion.

It follows now from Proposition 1 that for $f, g \in P_{0} F$ there is a unique elementary measure $p(f, g)$ (carried by $X_{0}$ ) of the $K$-representation generated by $V_{0}$. Consequently $V_{0}=\int z d F(z), F$ being a spectral measure.

(1) I take here the opportunity to express my thankss to Professor Glicksberg for making me known this proposition and its proof - W. M.

4 - Annales Polonicl Mathematicl XXV 
Applying the Sz.-Nagy-Dixmier theorem we infer that the following holds true:

Propositron 2. The part $\nabla_{0}$ is similar to a normal operator. If $K=1$, then $\nabla_{0}$ is normal.

Using the above proposition and the results of [12] we get easily the following

THEOREM 2. Suppose $X \subset C$ is a spectral set of $V \in L(H)$. Let $X=\bigcup_{k}^{s} X_{k}$ be the Bishop decomposition of $X$ relative to $R(X)$. Then $V=\underset{k \mid 0}{\stackrel{s}{m}} \nabla_{k}$, where $V_{k}$ has $X_{k}$ as a speotral set for $\eta_{0}>0$ and $V_{0}$ is normal with spectral measure carried by $\partial X$.

CoRolLARY 3. If $V$ is $\partial X$-pure (2), then $V_{0}=0$ in the above theorem.

We will now consider the partitions of spectral sets induced by Gleason parts of $R(X)$. In what follows the algebra $R(X)$ is viewed as a function algebra on $\partial X$, i.e. consisting of the restrictions of functions of $R(X)$ to $\partial X$. A result of Willzen [18], Th. 3.3, says that representing measures for points in a part $G$ for $R(X)$ have closed supports in $\vec{G}$ and represent this points for $R(\bar{G})$ as well. Since $R(X)$ is viewed as an algebra on $\partial X$, the representing measures for points in $G$ are carried by $\bar{G} \cap \partial X \subset \partial \bar{G}$.

Suppose $\dot{X}$ is a $K$-spectral set of $V \dot{\epsilon} L(H)$. Then by Theorem 2.1 of [13] $H=H^{\prime}+H^{\prime \prime}$ and $V=V_{G}+V_{S}$ (both sums direct), where $V_{G}$ $\epsilon L\left(H^{\prime}\right), \nabla_{S} \in L\left(H^{\prime \prime}\right)$ have $X$ as a $K$-spectral set; $V_{G}$ generates a $G$-continuous representation of $R(X)$, and $\nabla_{S}$ a $G$-singular one ( $\left.{ }^{3}\right)$. The representation generated by $V_{G}$ extends uniquelly to a $\partial \bar{G}$-supported $K$-representation of $\overline{(R(\bar{X}) \mid \bar{G})}$. Since in general $\overline{(R(X) \mid \bar{G})} \neq R(\bar{G})$, we cannot just conclude at this point that $\bar{G}$ is $K$-spectral for $\nabla_{G}$. If $G$ has a connected complement, then $R(\bar{G})=\overline{(R(\bar{X}) \mid \bar{G})}$ by Mergelyan theorem, which proves that in this special case $\bar{G}$ is $K$-spectral for $V_{G}{ }^{(4)}$. However, as proved by Sarason in [17], if $\dot{K}=1$ and $X$ has connected complement, then $\bar{G}$ is spectral for $\nabla_{G}$ provided $X$ is spectral for $V$. The key property (besides the essential dilatability of the representation generated by $V$ ) applied by Sarason was Proposition 13 of [17]. The proposition below is a generalization of this proposition ( $\left.{ }^{5}\right)$.

Proposition 3. Suppose $G$ is a Gleason part for $R(X)$ and let the measure $p \perp R(X)$. Then the G-continuous part $p_{G}$ of $p$ is orthogonal to $R(\bar{G})$.

(2) See [6], [11] and [17] for related matter.

(3) See [13] for definitions.

(4) The proof of this property, erronously suggested as applicable in a general case may bo found in [14].

(5) I would like to thanls here Professor I. Glicksberg for pointing out to me the enclosed proof of Proposition 3. It is much simpler than that of mine - W. M. 
Proof. The general M. and F. Riesz theorem of [8] yields that $p_{G} \perp R(X)$. Suppose $z_{0} \in X-\bar{G}$. It follows that $p_{G}$ is singular with respect to $M_{\ddot{z}_{0}}$ and if $m \in M_{\varepsilon_{0}}$, then

$$
q=\frac{1}{z-z_{0}} p_{Q}-m \int \frac{d p_{Q}}{z-z_{0}} \perp\left(C+\left(z-z_{0}\right) R(X)\right) .
$$

For any $z_{0} \in X, C+\left(z-z_{0}\right) R(X)(z \in X)$ is dense in $R(X)$, so $q \perp R(X)$ and its $M_{x_{0}}$-singular part $\frac{1}{z-z_{0}} p_{G} \perp R(X)$. Hence

$$
\hat{p}_{G}\left(z_{0}\right) \stackrel{\text { df }}{=} \int \frac{d p_{G}}{z-z_{0}}=0 \quad \text { for } z_{0} \in X-\bar{G} .
$$

Since $p_{G} \perp R(X), \hat{p}_{G}\left(z_{0}\right)=0$ for $z_{0} \notin X$ also, which completes the proof. As already noticed, the elementary measures of the representation of $R(X)$ generated by $\nabla_{G}$ being $G$-continuous are supported by $\partial \vec{G}$. If $p^{\prime}(f, g), p^{\prime \prime}(f, g)\left(f, g \in H^{\prime}\right)$ are two such measures, then $R(X) \perp p^{\prime}-p^{\prime \prime}$ $=\left(p^{\prime}-p^{\prime \prime}\right)_{G}$ and by Proposition $3, p^{\prime}-p^{\prime \prime} \perp R(\bar{G})$. It follows that

$$
\xi(u ; f, g)=\int_{\overline{\bar{G}}} u d p^{\prime}(f, g)=\int_{\bar{G}} u d p^{\prime \prime}(f, g)
$$

is a well defined functional for $u \in R(\bar{G})$, linear in $f$ and antilinear in $g$ and such that $|\xi(u ; f, g)| \leqslant K$ sup $|u|\|f|\||| \mid\|$. We conclude that there is linear mapping $T: R(\bar{G}) \rightarrow L\left(H^{\prime}\right)$ such that $\|T(u)\| \leqslant K\|u\|_{G}\left(\|u\|_{G}=\sup _{\bar{G}}|u|\right)$ and

$$
(T(u) f, g)=\int_{\bar{G}} u d p(f, g) \quad\left(u \in R(\bar{G}), f, g \in H^{\prime}\right)
$$

$p$ being elementary measure. In fact $\int_{\bar{G}} u d p(f, g)=\int_{\theta \bar{G}} u d p(f, g)$. Suppose now that $z_{0} \in X$ but $z_{0} \notin \bar{G}$ and consider the measure $p\left(f,\left(V_{Q}-z_{0} I\right)^{*} f\right)$ $\ll M_{\tilde{\varepsilon}}(\tilde{z} \epsilon G)$ for $f \in H^{\prime}$. Since $X-\bar{G}$ is a $F_{\sigma}$ set, the Forelli lemma ([6], Oh. II. 7.3, p. 43) proves that there is a norm bounded sequence of rational functions $u_{\nu} \in R(X)$ such that

$$
\begin{array}{ll}
u_{\nu}(z) \rightarrow 0 & \text { for } z \epsilon X-\bar{G}, \\
u_{\nu}(z) \rightarrow 1 & \text { a.e. } M_{\tilde{z}}(\tilde{z} \epsilon G) .
\end{array}
$$

We define

$$
r_{v}(z)=\frac{u_{v}(z)-u_{\nu}\left(z_{0}\right)}{z-z_{0}}
$$


Then $r_{\nu} \in R(X)$. Since $X$ is $K$-spectral for $\nabla_{G}$ with $\partial \vec{G}$-supported elementary measures, $T(r)=r\left(\nabla_{G}\right)$ for every polynomial $r$, which for $f \in H^{\prime}$ gives us

$$
\begin{aligned}
\left\langle r\left(\nabla_{G}\right)\left(u_{\nu}\left(\nabla_{G}\right)-u_{\nu}\left(z_{0}\right) I \mid f, f\right)\right. & =\left(\left(V_{G}-z_{0} I\right) r\left(V_{G)} r_{\nu}\left(\nabla_{G}\right) f, f\right)\right. \\
& =\int_{\bar{G}} r(z) r_{\nu}(z) d p_{z}\left(f,\left(V_{G}-z_{0} I\right)^{*} f\right) \stackrel{\mathrm{df}}{=} \cdot \eta_{\nu} .
\end{aligned}
$$

Since $z_{0} \notin \bar{G}$ and $u_{\nu}$ are norm bounded

$$
\left|r_{\nu}(z)\right| \leqslant \varrho, \quad \nu=1,2, \ldots, z \in \vec{G}
$$

for some finite constant $\varrho$. By dominated convergence and by (8) and (9) we have

$$
\eta_{v} \rightarrow \int_{\bar{G}} r(z) \frac{1}{z-z_{0}} d p_{z}\left(f,\left(V_{G}-z_{0} I\right)^{*} f\right) \stackrel{\mathrm{d} t}{=} \eta
$$

because $p\left(f,\left(V_{G}-z_{0} I\right)^{*} f\right)$ is $G$-continuous. The definition of $T(u)$ jields that

$$
\left.\eta=\dot{(T}\left(\frac{r}{z-z_{0}}\right) f,\left(\nabla_{G}-z_{0} I\right)^{*} f\right) .
$$

But $\eta_{\nu} \rightarrow\left(r\left(\nabla_{G}\right) f, f\right)$ by (8). Since $f$ is an arbitrary vector of $H^{\prime}$, we get that

$$
r\left(V_{G}\right)=\left(V_{G}-z_{0} I\right) T\left(\frac{r}{z-z_{0}}\right) .
$$

On the other hand, when considering for $f \in H^{\prime}$ the $G$-continuous elementary measure $p\left(\left(V_{G}-z_{0} I\right) f, f\right)$ we infer that

by (8) and (9). It follows that

$$
\begin{aligned}
\left(r\left(V_{G}\right)\left(u_{\nu}\left(V_{G}\right)-u_{\nu}\left(z_{0}\right) I\right) f, f\right) & =\int_{\bar{G}} r(z) r_{\nu}(z) d p_{z}\left(\left(\nabla_{G}-z_{0} I\right) f, f\right) \\
& \rightarrow\left(T\left(\frac{r}{z-z_{0}}\right)\left(V_{G}-z_{0} I\right) f, f\right)
\end{aligned}
$$

$$
r\left(V_{G}\right)=\left(V_{G}-z_{0} I\right) T\left(\frac{r}{z-z_{0}}\right) \text {. }
$$

Talking $r(z) \equiv 1$ we infer by (10) and (11) that $z_{0} \notin \mathrm{sp}\left(V_{G}\right)$ and

$$
T\left(\frac{1}{z-z_{0}}\right)=\left(\nabla_{G}-z_{0} I\right)^{-1}
$$

This together with (10) and (11) shows that for $z_{0} \in X-\bar{G}$

$$
T\left(\frac{r}{z-z_{0}}\right)=r\left(V_{G}\right)\left(V_{G}-z_{0} I\right)^{-1} .
$$

Since $T(u)=u\left(V_{G}\right)$ for rational $u$ having poles off $X,(12)$ holds for every $z_{0} \notin \bar{G}$. 
Let $u$ be a rational function having merely simple poles $z_{1}, \ldots, z_{m}$ all lying in $\vec{Q}$. Then $u$ may be written in the form

$$
u(z)=\sum_{i \mid 1}^{m} \frac{u_{i}(z)}{z-z_{i}}
$$

with suitable polynomials $u_{i}$. The linearity of the mapping $T$ implies that

which by (12) gives us

$$
T(u)=\sum_{i \mid 1}^{m} T\left(\frac{u_{i}}{z-z_{i}}\right)
$$

$$
T(u)=\sum_{i \mid 1}^{m} u_{i}\left(\nabla_{G}\right)\left(\nabla_{G}-z_{i} I\right)^{-1}=\sum_{i \mid 1}^{m}\left(\nabla_{G}-z_{i} I\right)^{-1} u_{i}\left(V_{G}\right)=u\left(\nabla_{G}\right) .
$$

Since $\|T(u)\| \leqslant K\|u\|_{\alpha}, \bar{G}$ is a $K$-spectral set of $\nabla_{G}$, because such $u$ are dense in $R(X)$. Summing up we get the following

THeorem 3. Suppose that $G$ is the Gleason part for $R(X)$. If $X$ is a $K$ speotral set of $\nabla \in L(H)$, then $\vec{G}$ is a $K$-spootral set of $\nabla_{G} \in L\left(H^{\prime}\right)$.

Combining Theorem 3 with Theorem 1, Theorem 3 of [14] we get the following

THEOREM 4. Suppose the compaot $X \subset C$ is a spectral set of $V \in L(H)$. Let $G_{i}(i=1,2, \ldots)$ be the sequenoe of all non-peate point parts for $R(X)$. Then

where:

$$
V=\stackrel{s}{\oplus} V_{i} \oplus V_{0} \quad(s \leqslant+\infty),
$$

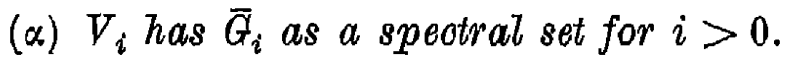

( $\beta$ ) The representation of $R\left(\bar{G}_{i}\right)$ generated by $V_{i}(i>0)$ has elementary measures absolutely continuous with respect to $M_{z}$ for $z \in G_{i}$.

$(\gamma) \nabla_{0}$ is a normal operator with spectrum carried by $\partial X$. Moreover, $\nabla_{0}=V_{0}^{\prime} \oplus V_{0}^{\prime \prime}$, where $V_{0}^{\prime}$ is normal having completely singular speotral measure and $V_{0}^{\prime \prime}=\oplus_{i} z_{l} I_{l}\left(I_{l}=i\right.$ dentity in suitable $\left.H_{l}\right)$, where $z_{l}$ 'ranges over the set of all peat points for $R(\dot{X})$.

Both Theorem 3 and Theorem 4. have been proved in a completely dilation free way. Theorem 4 generalizes the Theorem 1 of Sarason paper [17], which concerned $X$ having connected complement. Any $V$ having such $X$ as a spectral set generates a uniquelly $\partial X$-dilatable representation of $R(X)$ (see [4] and also [10]). This fact has been used essentially in [17].

COROLLARY 4. If $V$ has no non-zero normal part with spectrum on $\partial X$, then simply $V=\stackrel{s}{\oplus} V_{i \mid 1}$. 


\section{References}

[1] A, Browder, Introduction to funotion algebras, New York 1969.

[2] R. G. Douglas, On operators similar to normal operators, Rev. Math. Pures et Appl. (Roumaine) 2, Tome XIV (1969), p. 193 - 197.

[3] J. Dixmier, Les moyennes invariantes dans les semi-groupes et leurs applioatione, Acta Sci. Math. (Szeged) 12 (1950), p. 213-227.

[4] C. Foies, Oertain applioatione of speotral sets, Studii si cercetari mat. 10 (1950), p. 365-401 (in Roumanian).

[5] - and I. Suciu, Srego measures, Rev. Roumaine Math. Pure Appl. 11 (1966), p. $147-160$.

[6] T. W. Gamelin, Uniform algebras, Englewood Cliffs, N. J. 1968.

[7] I. Glicks berg, Measures orthogonal to algebras and sets of antisymmetry, T.A.M.S. 105 (1969), p. $415-435$.

[8] - The abstraot $P$. and $M$. Riesz theorem, J. Funet. An. 1 (1967), p. 109-122.

[9] - Dominant representing measures and rational approximation, T.A.M.S. 130 (1968), p. $425-462$.

[10] A. Lebow, On von Neumann's theory of spectral sets, J. Math. Anal. Appl. 7, No. 1 (1963), p. 64-90.

[11] W. Mlak, A note on Sregö type properties of semi-speotral measures, Studia Math. 31 (1968), p. 241 - 251.

[12] - Dooomposition of operator-valued representations of funotion algebras, ibidem 36 (1970), p. $111-123$.

[13] - Decompositions and entensions of operator-valued representations of funotion. algebra8, Acta Sci. Math. (Szeged)' 30 (1969), Fasc. 3-4, p. 181-193.

[14] - Absolutely continuous operator-valued representations of funotion algebras, Bull. Acad. Sci. Polon. 17 (9) (1969), p. 547-550.

[15] J. von Neumann, Eine Speltraltheorie für allgemeine Operatoren eines unitdren Raumes, Math. Nachr. 4 (1951), p. 258-281.

[16] R. R. Phelps, Leotures on Choquet's theorem, Van Nostrand Math. St., Prin. ceton, N. Y. 1966.

[17] D. Sarason, On speotral sets having oonneoted complement, Aota Sci. Math. (Szeged) (1965) 26, 3-4, p. $289-299$.

[18] D. R. Wilken, The support of representing measures for $R(X)$, Pacific J. Math. 26 (3), (1868), p. $621-626$. 